\title{
Commentary: Running the cardiovascular intensive care unit-Should surgeons be cut?
}

\author{
Vaibhav Gupta, MD
}

\footnotetext{
From the Division of General Surgery, Department of Surgery and Institute of Health Policy, Management, and Evaluation, University of Toronto, Toronto, Ontario, Canada.

Disclosures: Author has nothing to disclose with regard to commercial support.

Received for publication April 26, 2019; accepted for publication April 29, 2019; available ahead of print June 20, 2019.

Address for reprints: Vaibhav Gupta, MD, Sunnybrook Health Sciences Centre K3W-15, 2075 Bayview Ave, Toronto, Ontario M4N 3M5, Canada (E-mail: Vaibhav.Gupta@mail.utoronto.ca).

J Thorac Cardiovasc Surg 2020;159:1392

$0022-5223 / \$ 36.00$

Copyright (c) 2019 by The American Association for Thoracic Surgery

https://doi.org/10.1016/j.jtcvs.2019.04.107
}

In this issue of the Journal, Lim and colleagues ${ }^{1}$ from Korea investigate the relationship between different physician staffing models in a cardiovascular intensive care unit (ICU) and short-term outcomes, including ICU and hospital stays, infection and transfusion rates, ICU readmission, and mortality. The study analyzed 4038 patients with a historical control design in a 4-year period: "normal-intensity" staffing from 2013 through 2014 and "high-intensity" staffing from 2015 through 2016. Given the challenge of creating a randomized, controlled trial to study this question, a historical control design encompassing a relatively short period seems fair. Lim and colleagues ${ }^{1}$ used a propensity score-matched analysis to adjust for confounders. The main distinction between normal- and high-intensity staffing in this study was based on whether the majority of care was provided by the primary physician (cardiac surgeon or surgical resident) or an intensivist (cardiac surgeon with critical care training). Some readers may be more familiar with the terms "open" versus "closed" ICU for this model, but Lim and colleagues ${ }^{1}$ used "intensity" terminology according to the definitions created by the Committee on Manpower for Pulmonary and Critical Care Societies. $^{2}$

Overall, there was no meaningful difference in length of stay (ICU or hospital stay) or mortality (ICU, in-hospital, or 30-day mortality) between the groups. With the intensivistmanaged cardiovascular ICU, Lim and colleagues ${ }^{1}$ found lower blood transfusion, infection, and ICU readmission rates. They observed that one of the main differences with high-intensity staffing was that the intensivists followed the unit's standard protocols more strictly, whereas management by cardiac surgeons (normal intensity) was influenced by their personal preferences. Lim and colleagues ${ }^{1}$ hypothesize that this may have driven some of the improved outcomes. This would be in line with the ICU and surgical literature, which shows that standardized management protocols can lead to better care.

In my view, there are 2 conclusions to draw from this study. First, outcomes are improved when patients receive

\section{References}

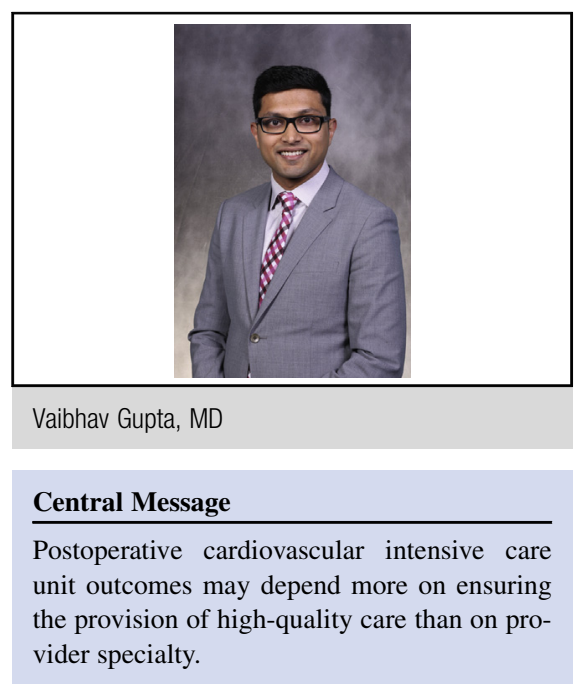

See Article page 1382.

more focused care from the physician team. Care in the normal-intensity group was largely provided by surgical residents, who may have other clinical responsibilities in addition to managing postoperative patients in the cardiovascular ICU. It is not surprising that outcomes would be better when there is a specific physician assigned to the unit. Second, adherence to standardized management protocols improves outcomes. I wonder whether there would have been any difference between the groups had physician presence within the unit been similar and better education was provided to the cardiac surgery residents or attendings about following the institution's established ICU protocols. It seems that the difference between the groups in this study was driven by the second group receiving more highquality, evidence-based care, and this is what we need to focus on providing. If a surgical service does not have the capacity to provide this in the cardiovascular ICU, an intensivist-run unit may be the solution.

1. Lim JY, Kang PJ, Kim JB, Jung SH, Choo SJ, Chung CH, et al. Influence of a highintensity staffing model in a cardiac surgery intensive care unit on postoperative clinical outcomes. J Thorac Cardiovasc Surg. 2020;159:1382-9.

2. Angus DC, Shorr AF, White A, Dremsizov TT, Schmitz RJ, Kelley MA, Committee on Manpower for Pulmonary and Critical Care Societies (COMPACCS). Critical care delivery in the United States: distribution of services and compliance with Leapfrog recommendations. Crit Care Med. 2006;34:1016-24. 